\title{
Hinged External Fixation of the Elbow: Optimal Axis Alignment to Minimize Motion Resistance
}

\author{
Steven M. Madey, $* \dagger$ Michael Bottlang, $\doteqdot$ Curtis M. Steyers, \\ $\ddagger$ John L. Marsh, and $\dagger \ddagger$ Thomas D. Brown
}

Department of Trauma Surgery, Legacy Emanuel Hospital, Portland, Oregon, U.S.A.; *Biomechanics Laboratory,

Legacy Health System, Portland, Oregon, U.S.A.; and †Department of Biomedical Engineering and $\doteqdot$ Department of Orthopaedic Surgery, University of Iowa, Iowa City, Iowa, U.S.A.

Objective: To establish an optimal single hinge axis position for application of hinged external fixation to the elbow joint.

Design: Cadaveric biomechanical investigation.

Setting: A customized motion transducer applied passive elbow motion to six cadaveric upper extremities. The instant rotation axis of the humero-ulnar articulation was determined from threedimensional kinematic data acquired by an electromagnetic motion tracking system. For each specimen, an optimal fixator hinge position was calculated from these motion data.

Intervention: A prototype articulated external fixator was applied to the elbow, first with its hinge aligned along the computed optimal position. Then the fixator was mounted in sixteen distinct off-axis positions.

Main Outcome Measure: Additional resistance to joint motion (in terms of energy) corresponding to deliberately introduced amounts of relative malalignment between the optimal elbow axis and the actual fixator hinge axis.

Results: Aligning the fixator hinge along the optimized axis position resulted in a minimal amount of energy ( 0.15 joules) needed to rotate the elbow through a prescribed range of motion. Malpositioning the hinge by ten millimeters caused up to ten times that amount of motion resistance.

Conclusions: An optimal fixator hinge position can be determined to minimize the increase in motion resistance due to fixator application. The severely increased motion resistance associated with small amounts of malalignment between the fixator hinge and the anatomic elbow axis suggests the need for highly accurate fixator hinge application.

Key Words: External fixation, Hinged fixation, Elbow kinematics, Fixator alignment, Screw displacement axis.
Since Volkov and Oganesian's original description in 1975 (34), several different models of hinged elbow external fixation have been described for the treatment of complex acute and chronic elbow disorders (11,13,24,34). Development of these fixators stems from the attempt to preserve both elbow stability and motion in specific elbow disorders that historically have responded poorly to treatment $(7,14,28)$. Kinematically the humero-ulnar articulation approximates a simple hinge joint $(8,11,12,15,18,31)$. Therefore, it seems logical that recreating the anatomic axis of an injured elbow with an appropriately positioned hinged

Accepted April 19, 1999.

Address correspondence and reprint requests to Dr. Michael Bottlang, Biomechanics Laboratory, Legacy Health System, 1225 Northeast 2nd Avenue, Portland, OR 97140, U.S.A.

Financial support for this project was provided by a grant from EBI Medical Systems Inc., Parsippany, New Jersey. The authors have received nothing of value.

Subsequent to submission of this manuscript for publication, EBI Medical Systems Inc. and the University of Iowa Hospital and Clinics applied for a patent on the hinged elbow fixator described herein. Michael Bottlang and John L. Marsh were named in the patent application. As of November 1999, a patent had not been granted.

The device that is the subject of this manuscript is not FDA-approved and is not commercially available in the United States. external fixator would allow early mobilization under load shielding of the articulation, with potential for improving the functional outcome.

Previous studies of elbow external fixation have corroborated this assumption $(9,16,24,36)$. However, most of these studies also identified problems with stiffness, persistent elbow instability, pin loosening, and pin breakage. Such problems may be related to off-axis positioning of the fixator hinge with respect to the anatomic elbow axis. Presently there is a lack of adequate descriptions of methods for accurately aligning the fixator hinge with the rotation axis of the elbow joint. We hypothesize that, if physiologic motion behavior is to be obtained, there is little room for error in aligning a hinged fixator on-axis in respect with the elbow. Thus, we attempted to quantify the positional effect of a hinged fixator on the induced motion resistance in a cadaveric elbow system.

\section{MATERIALS AND METHODS}

\section{Experimental Set-up}

Six fresh-frozen cadaveric upper extremities with no radiographic or visual evidence of degenerative joint 


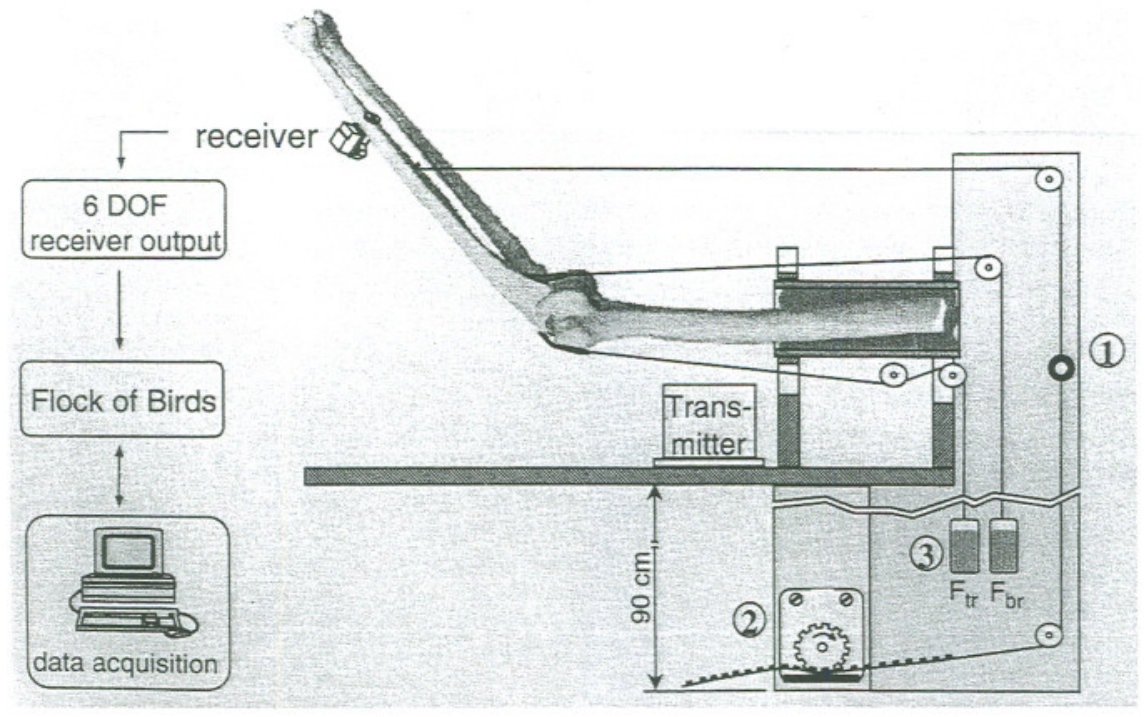

FIG. 1. Test set-up (drawing not to scale) for the application of minimally restrained elbow motion; shown for elbow rotation toward flexion. (1) Custom load cell. (2) DC motor. (3) Counterweights. disease (osteophytes, cartilage destruction) were amputated at the mid-humeral shaft and disarticulated at the radiocarpal joint. The skin, subcutaneous tissues, and muscles were excised, whereas the joint capsule, ligaments, and musculotendinous insertions of the brachialis and triceps were retained. The mid-diaphysis of the humerus was secured with polymethyl methacrylate in a Plexiglas tube, which in turn was rigidly affixed to a specially designed elbow motion applicator (Fig. 1). This experimental set-up applied flexion or extension moments to the elbow joint in a minimally constraining manner via a cable attached to the ulna at a point 150 millimeters distal to a line through the epicondyles. The resulting cable tension was continuously recorded with a custom load cell (1) in Fig. 1). A direct current (DC) motor (2) in Fig. 1) with an integrated rotation encoder generated the force necessary to rotate the elbow through its range of motion (ROM). The output of the rotation encoder allowed continuous assessment of the apparent elbow flexion angle. Counterweights (3) in Fig. 1) were attached to the musculotendinous insertions via anatomically aligned cables in order to simulate a constant applied brachialis force, $\mathrm{F}_{\mathrm{br}}$ (ten newtons), and triceps force, $\mathrm{F}_{\mathrm{tr}}$ (ten newtons). These muscle forces were simulated to ensure continuous joint compression force between the articular surfaces.

\section{Elbow Kinematics}

The kinematics of elbow joint rotation were recorded with a DC electromagnetic motion tracking system ("Flock of Birds," model 6DFOB, Ascension Technology Corp., Burlington, VT, U.S.A.). This instrument traced the motion of a receiver coil triad with respect to a transmitter, and operated at a sampling rate of fifty-eight hertz. The transmitter was rigidly connected to the humerus. The receiver was attached to the mid-diaphysis of the ulna. The receiver output completely described the three-dimensional kinematics of the ulna with respect to the humerus, with translational and rotational accuracy of 0.5 millimeters and 0.1 degree, respectively (17). To avoid distortion of the motion tracking recordings due to interference from nearby ferromagnetic objects $(5,17)$, the entire experimental setup was built from nonmagnetic materials (e.g., Plexiglas, fiberglass), and the DC motor was located remotely (ninety centimeters) from the transmitter. The electromagnetic motion tracking data were used to compute the instant rotation axis of the elbow joint, described in terms of rigid body screw displacement axes (SDAs). SDAs were calculated for incremental rotations of 5 degrees, using Beggs's (3) algorithm in combination with a customized source data smoothing procedure (5). For each specimen, a "bestfit" SDA was defined by averaging all SDAs obtained over the entire ROM. Finally, the average SDA was physically marked in each specimen to enable fluoroscopic visualization of the SDA location, as well as to record the radiographic appearance of the elbow joint from the viewpoint of an x-ray beam siting precisely along the average SDA. Anteroposterior radiographs were used to obtain the longitudinal axis of the humerus and ulna by fitting a line to the geometric center of the diaphyseal shaft.

\section{Hinged Monolateral External Fixator}

A prototype hinged monolateral external fixator for the elbow was designed to allow positioning of the fixator hinge independent of anchorage pin position. This fixator was free of any ferromagnetic components. The primary body elements of the modular fixator were carbon fiber rods. These were interconnected via aluminum clamps to form a rigid double-bar construct proximal and distal to the fixator hinge (Fig. 2). Initially, this modular fixator was applied to the elbow with its hinge axis aligned along the calculated best-fit axis. This was achieved by calculating the intersection points (A, B) of the best-fit SDA with two planes medial and lateral to the elbow (Fig. 3). A hinge alignment fixture was then placed between these two intersection points to establish an accurate fixator hinge position in three-space. 
FIG. 2. Experimental hinged monolateral external fixator for the elbow. The fixator hinge position is adjustable, independent of the anchorage pin positions.

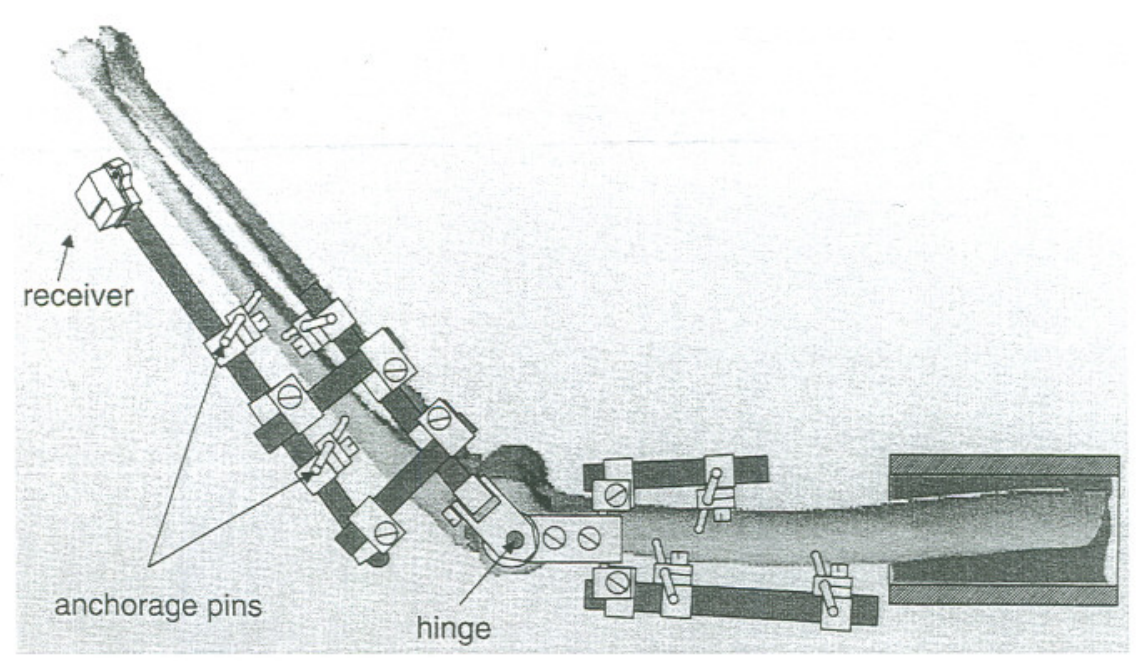

Guided by the alignment fixture, the fixator was applied with the elbow in 60 degrees of flexion (defined as our "neutral position"). Then, starting with the elbow in this neutral position, the moment required to rotate the elbow toward flexion and then extension was determined from the load cell recordings. Next, to assess the effect of hinge malalignment on joint kinematics, the fixator hinge was mounted in each of sixteen distinct off-axis positions (Fig. 4). The hinge axis was translated five and ten millimeters anteriorly/ posteriorly/proximally/distally, and then angulated in 5 degrees and 10 degrees of eversion/inversion/internal rotation/external rotation, where the angulation occurred around a parasagittal plane, centered between the epicondyles. Again, the moment history during flexion and extension was determined for each of the off-axis positions. To condense the large data set, the energy necessary to rotate the elbow one time through an 86 degree ROM, from 23 degrees to 109 degrees, was calculated from the moment versus angular displacement data by integrating the area under the moment curves over the 86 degree ROM. This ROM was the largest achievable moment data range common to all specimens, because impingement of the fixator bars/clamps with the adjustable hinge components occurred in some of the hinge off-axis positions. To isolate the energy required to rotate the elbow, the moment caused by the counterweights was subtracted from all moment data before the energy was calculated.

The outcome variable (energy) was statistically analyzed via a two-sided two-sample Student's $t$ test for unequal variances at a significance level of 0.05 . All fixator applications (on-axis as well as sixteen off-axis hinge placements) were analyzed with respect to the following hypothesis $\left(\mathrm{H}_{0}\right)$ : compared with the natural elbow, fixator application does not cause a statistically significant energy increase.

\section{RESULTS}

The pathway of the SDA for an elbow moving in flexion, through a ROM from 30 to 127 degrees of flexion, is shown in Figure 5. For purposes of visualization, the SDA excursion is projected, with scale exaggeration, onto intercept planes located 200 millimeters medially and laterally from the geometric center of the trochlea. The limits of excursion of instantaneous SDA orientation in the horizontal and frontal planes, respectively, were $\alpha_{\mathrm{h}}=5.7 \pm$ 2.2 degrees and $\alpha_{\mathrm{f}}=2.6 \pm 1.0$ degrees (mean \pm standard deviation). The corresponding limits of SDA translation were $t_{h}=1.4 \pm 0.3$ millimeters and $t_{f}=2.0 \pm 0.6$ millimeters. All instantaneous SDAs nearly intersected at the medial facet of the trochlea, the minimal SDA dispersion occurring at a site $20 \pm 4.4$ millimeters medial to a parasagittal plane centered between the medial border of the trochlear facet and the lateral border of the capitellum (Fig. 6). The computed best-fit SDA penetrated the anteroinferior aspect of the medial epicondyle, the center of the trochlea, and the center of the projection of the capitellum onto a parasagittal plane. For a fully extended elbow

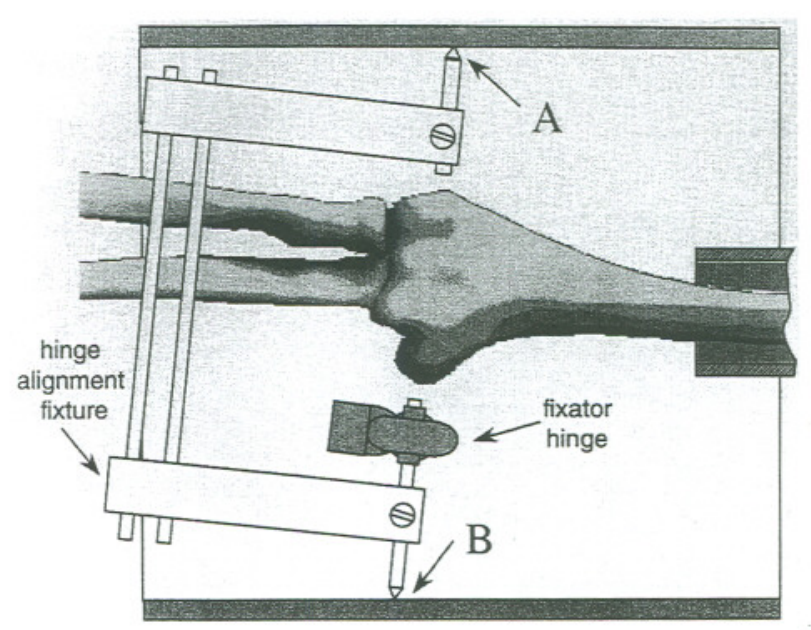

FIG. 3. Method for accurate placement of the fixator hinge. Points $A$ and $B$ are the intersection points of the best-fit screw displacement axis (SDA) with planes medial and lateral to the elbow. 


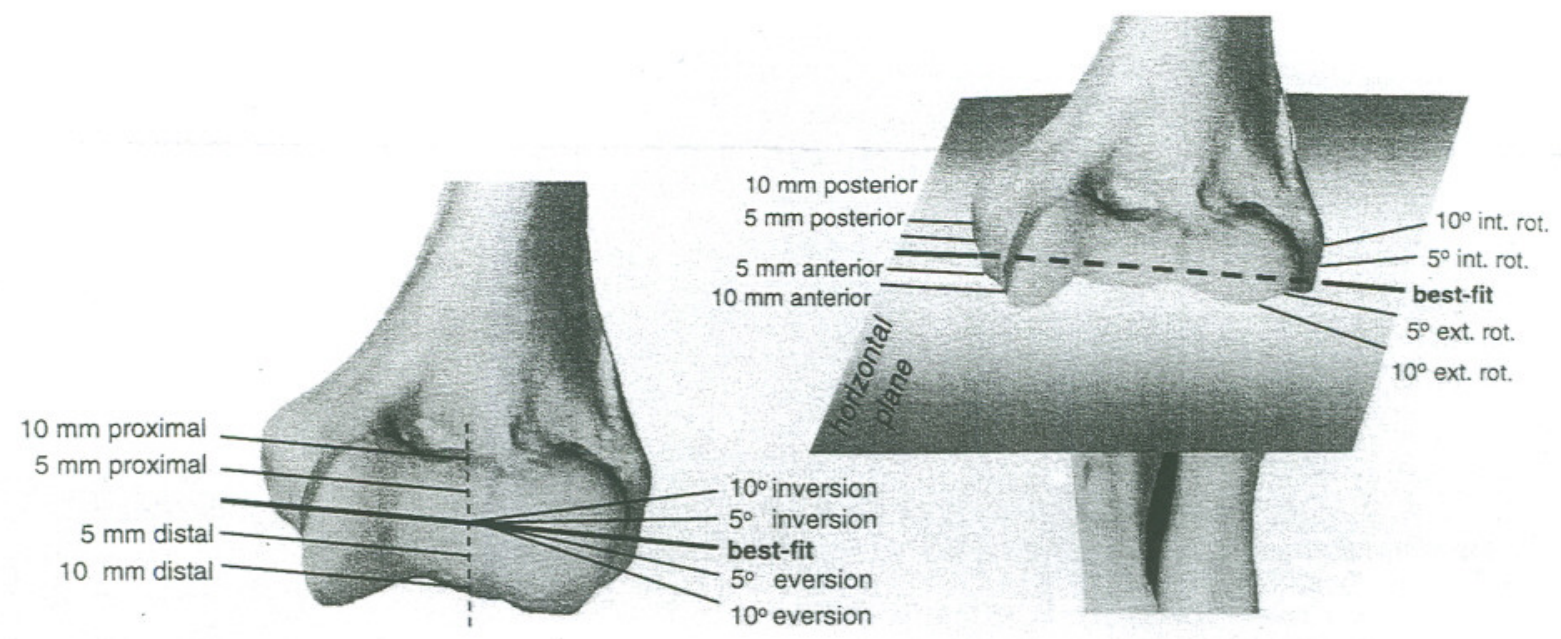

FIG. 4. The sixteen off-axis positions (relative to the best-fit position) for application of the external fixator hinge.

viewed in the frontal plane, the best-fit SDA formed angles of $86.1 \pm 2.5$ degrees and $85.4 \pm 3.8$ degrees with the longitudinal axes of the humerus and ulna, respectively.

Application of the external fixator to the elbow constrained motion of the elbow joint to be purely rotation around the single fixed hinge axis of the fixator. The amount of energy needed to rotate the natural elbow (no fixator applied) through an 86 degree ROM (from 23 to 109 degrees of flexion) was less than 0.02 joules. Applying the fixator with its hinge precisely aligned along the computed best-fit axis produced slightly more motion resistance. However, the corresponding average energy of $0.15 \pm$ 0.14 joules was not statistically significantly higher as compared with the natural elbow. Any of the sixteen dis- tinct off-axis hinge locations resulted in statistically significantly higher energy values as compared with the normal, unconstrained elbow joint. Therefore, $\mathrm{H}_{0}$ had to be rejected for every off-axis fixator application, verifying that the computed best-fit location established an optimal hinge position that involved minimal increase in motion resistance (Fig. 7A).

There was no correlation between the direction of malalignment and the energy increase. Hinge off-axis translations of five millimeters required on average 4.0 times the energy of the best-fit hinge position. Off-axis translations of ten millimeters resulted on average in 8.2fold higher (maximum tenfold) energy values. This was true for off-axis translations in all four directions. Hinge

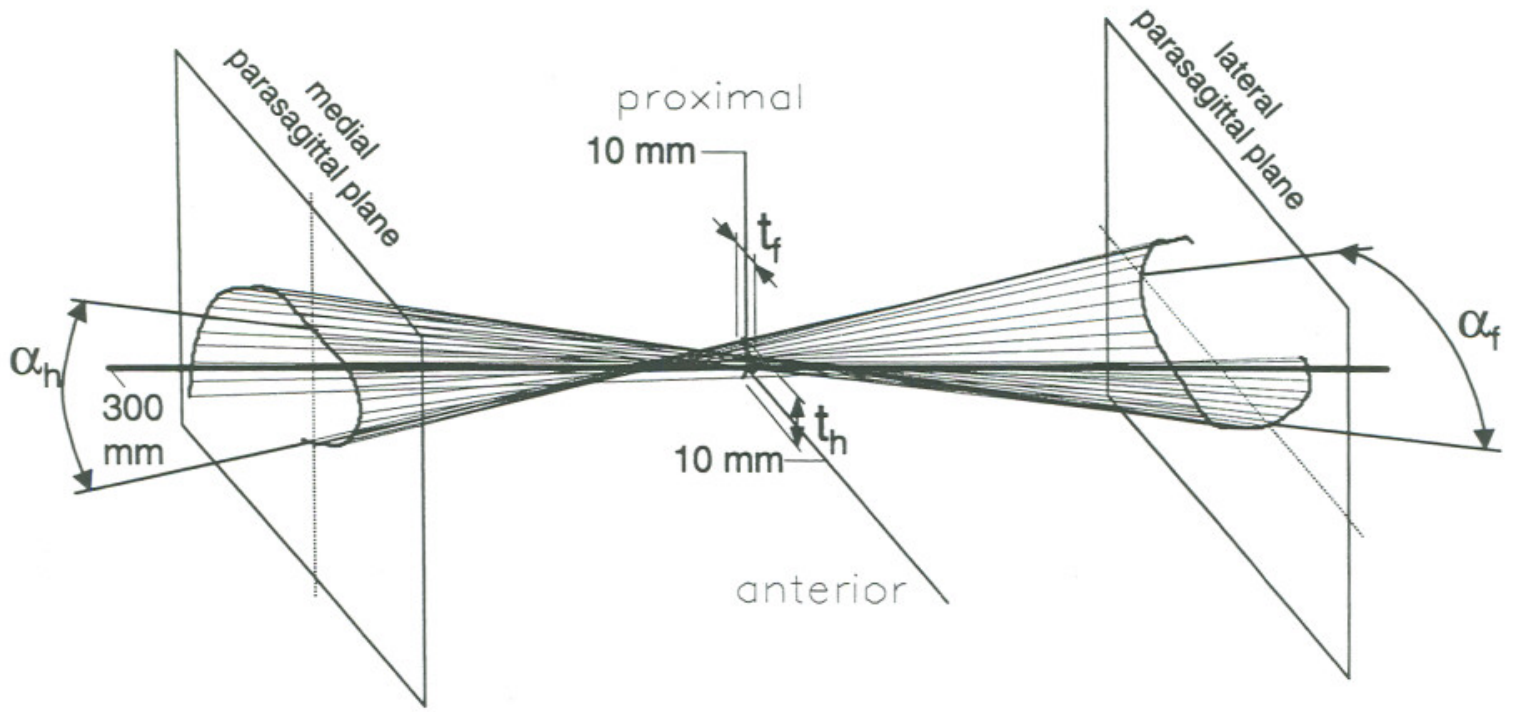

FIG. 5. Pathway of SDA with respect to the humerus. For purpose of visualization, the SDA excursion is exaggerated by tracing the projection onto parasagittal planes located 200 millimeters medial and lateral of the trochlea. Note the large scaling difference within versus perpendicular to these intercept planes. 


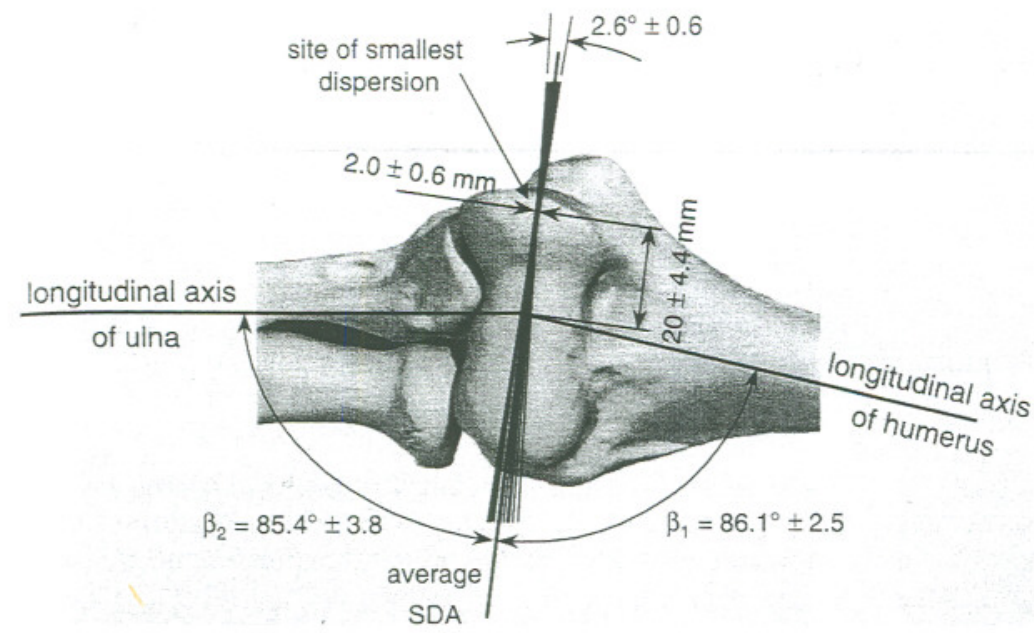

FIG. 6. Location of the computed best-fit SDA with respect to the elbow joint, in the frontal plane. SDA excursion is exaggerated by a factor of 2.0 to help visualize the site of the smallest SDA dispersion. off-axis orientations of 5 degrees required an average 3.7fold increase in the motion energy over the best-fit hinge position (Fig. 7B). Off-axis orientations of 10 degrees averaged a 7.1-fold energy increase, and a maximum of an 8.7-fold increase.

\section{DISCUSSION}

The elbow is a relatively stable joint, owing to its osseous geometry and ligamentous restraints $(2,13,15,18,19,20$, $21,23,25,29,32,35)$. However, there are a number of clinical situations in which these stabilizers are lost, with resultant elbow instability $(1,4,7,10,28)$. These include, but are not restricted to: elbow dislocation with unstable reduction, medial collateral ligament injury with radial head fracture, comminuted olecranon and/or distal intra-articu- lar humerus fractures, and surgical release of a contracted or ankylosed joint. Attempts at stabilization in these situations often involve joint immobilization, with resultant joint stiffness or persistent instability $(7,14,28)$. Because the motion of the elbow joint approximates that of a simple hinge, rigid fixation of an unstable elbow with a constrained hinge aligned along the best-fit axis provides the advantage of early "physiologic" motion and preservation of elbow stability, offering the potential for a greater long-term functional ROM.

All clinical studies show favorable outcomes using hinged external elbow fixation devices for specific, complex elbow disorders. With this custom fixator, Volkov and Oganesian produced ROM gains of 40 to 120 degrees in a subgroup of twenty-four ankylosed joints in a series of thirty-eight patients with complex elbow disorders (34).
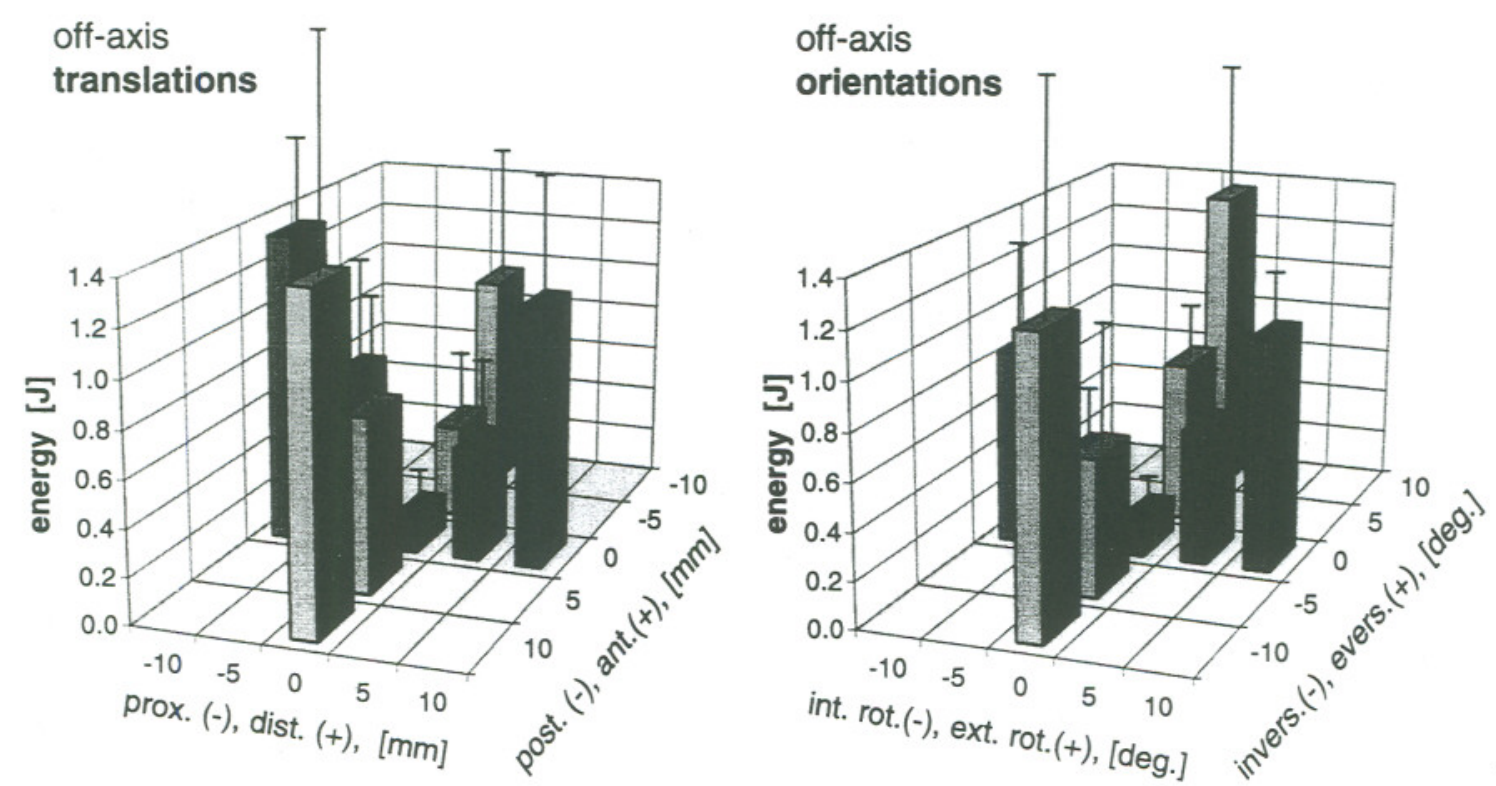

FIG. 7. Energy required to rotate the elbow through an 86 degree ROM ( 23 to 109 degrees) for the sixteen hinge off-axis translations (A) and orientations (B). 

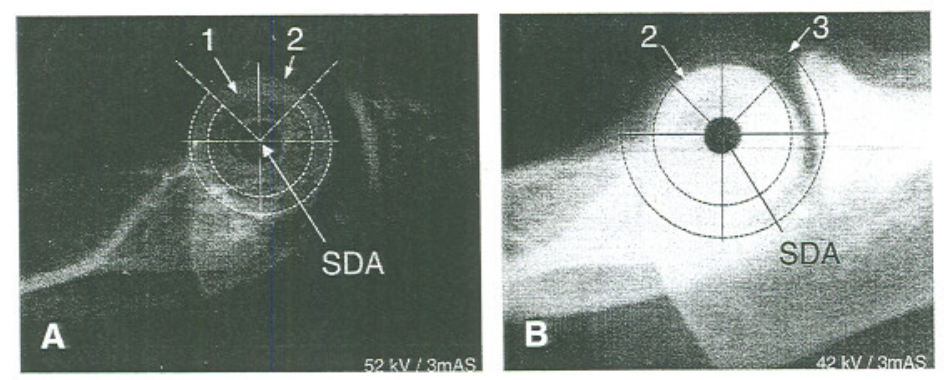

FIG. 8. Radiographic appearance of the elbow, with an $\mathrm{x}$-ray beam siting precisely along the best-fit SDA. The rotation axis lies in the center of three concentric shadows, formed by (1) the bottom of the trochlear sulcus, (2) the periphery of the capitellum, and (3) the medial facet of the trochlea. A: The shadow formed by the bottom of the trochlear sulcus (1) is visible at a high $\mathrm{x}$-ray excitation voltage (fifty-two kilovolts). B: In contrast, the shadow formed by the medial facet of the trochlea (3) is visible at a low $\mathrm{x}$-ray excitation voltage (forty-two kilovolts).

Half of the twenty-four patients experienced ROM gains greater than 70 degrees. Using his own device, Morrey reported average ROM gains of 55 degrees in a series of fifty-eight patients with severe contractures (24). Also using the Morrey device, Cobb and Morrey reported a significant increase in satisfactory outcome (an increase of 55 degrees of ROM) in seven patients with persistent instability after posterior fracture-dislocation (8). With the "Compass Hinge" (Smith \& Nephew, Memphis, TN, U.S.A.) McKee et al. showed good or excellent results (106 degrees average ROM) in nine out of twelve patients treated for acute complex instability (16). Wyrsch et al. reported sixteen out of twenty good or excellent results in treatment of contracture and instability (36). Volkov and Oganesian and Morrey reported few complications, limited to small numbers of superficial and deep infections and persistent instability $(24,34)$. However, Cobb and Morrey reported problems with persistent instability and pin migration in a third of their patients (9). McKee et al. reported a 25 percent incidence of either persistent instability, dislocation, or broken pins; in addition, one-half of their patients required remanipulation for stiffness (16). Wyrsch et al. reported two broken pins in their series of twenty patients (36).

Constraining the motion of the elbow joint to be purely rotation around the fixed hinge of an external fixator leads to an increase in the energy needed to rotate the joint through its ROM. By using the three-dimensional motion data, we were able to compute an optimal hinge position, for which this energy increase was minimal. The large additional resistance to motion accompanying a malpositioned fixator axis suggests the development of untoward intra-articular forces that could pose several problems in the clinical setting. In an acute unstable situation with loss of soft tissue and/or bony restraints, malalignment of the fixator hinge would impose abnormal joint kinematics, with resultant incongruous articulation and/or joint instability. In addition, as healing progresses to create a more constrained situation, increased stress would be transferred to the pins and the pin-bone interface. This may account for the clinical problems of pin loosening, pin breakage, and persistent instability.

In this study the effect of fixator hinge malalignment was quantified, with the results emphasizing the importance of correct fixator hinge placement, so as to achieve a motion path in concert with the natural motion of the joint. For clinical application, two issues merit attention. First, the fix- ator pins should not dictate the position of the fixator hinge. Instead, the fixator design must enable unconstrained alignment of the hinge, independent of pin position. Second, our findings underscore the need for a highly accurate intraoperative "axis-finding" technique.

In present clinical practice, elbow axis location is guided by anatomic or radiographic landmarks. Morrey described the axis as penetrating the inferior and anterior aspect of the medial epicondyle and the center of the projection of the capitellum (tubercle of lateral collateral ligament origin) (24). London described the use of concentric arcs on radiographs, corresponding to the bottom of the trochlear sulcus, the periphery of the capitellum, and the medial facet of the trochlea (15). Although we could verify London's radiographic landmarks (Fig. 8), the referenced concentric shadows were difficult to identify and were of inconsistent visibility. Fixator hinge placement based on such methods has a significant margin of error for several reasons. It is difficult to precisely identify nonspecific anatomic landmarks located some distance from the epicondyles. Also, there are problems in obtaining true lateral radiographs in the operating room, Kirschner wire migration occurs when trying to place an "axis pin," and it is often difficult to define normal anatomy in pathologic conditions. Furthermore, contemporary articulated elbow fixator designs do not accommodate hinge position adjustment after the fixator pins have been inserted.

The results presented soundly demonstrate that there is little room for error in aligning the fixator hinge with the elbow rotation axis. Implementation of these findings for the advancement of hinged external elbow fixation requires further research to establish an accurate intraoperative axis detection procedure and fixator hardware that allows flexible hinge positioning, independent of the fixator pins.

Acknowledgments: We are grateful to Dr. Douglas R. Pedersen for his technical assistance. Financial support for this project was provided by a grant from EBI Medical Systems Inc., Parsippany, New Jersey.

\section{REFERENCES}

1. Ackermann G, Jupiter JB. Non-union of fractures of the distal end of the humerus. J Bone Joint Surg [Am] 1988;70A:75-83.

2. An KN, Morrey BF, Chao EYS. The effect of partial removal of proximal ulna on elbow constraint. Clin Orthop Rel Res 1986;209: 270-279.

3. Beggs JS. Kinematics. Berlin, Springer-Verlag, 1983. 
4. Bennet JB, Green MS, Tullos HS. Surgical management of chronic medial elbow instability. Clin Orthop Rel Res 1992;278:62-68.

5. Bottlang M, Marsh JL, Brown TD. Factors influencing accuracy of screw displacement axis detection with a DC-based electromagnetic motion tracking system. J Biomech Eng 1998;120:431-435.

6. Bottlang M, Madey SM, Steyers CM, Marsh JL, Brown TD. Assessment of elbow joint kinematics in passive motion via electromagnetic motion tracking. J Orthop Res 1999 (in press).

7. Broberg MA, Morrey BF. Results of treatment of fracture-dislocations of the elbow. Clin Orthop Rel Res 1987;216:109-199.

8. Chao EYS, Morrey BF. Three-dimensional rotation of the elbow. J Biomech 1978;11:57-73.

9. Cobb TK, Morrey BF. Use of distraction arthroplasty in unstable fracture dislocations of the elbow. Clin Orthop Rel Res 1995;312: 201-210.

10. Davidson PA, Moseley BJ, Tullos HS. Radial head fractures. Clin Orthop Rel Res 1994;297:224-230.

11. Deland JT, Garg A, Walker PS. Biomechanical basis for elbow hinge-distractor design. Clin Orthop Rel Res 1987;215:303-312.

12. Fischer G. Handbuch der Anatomie und Mechanik der Gelenke, unter Beruecksichtigung der bewegenden Muskeln, Vol. 2. Jena, Germany, 1911.

13. Hotchkiss RN, Weiland A. Valgus stability of the elbow. J Orthop Res 1987;5:372-377.

14. Josefsson PO, Gentz OJ, Wendenberg B. Dislocation of the elbow and intraarticular fractures. Clin Orthop Rel Res 1989;246:126-130.

15. London JT. Kinematics of the elbow. J Bone Joint Surg [Am] 1981;63A:529-535.

16. McKee MD, Richards RR, Patterson SD, King JW, Jupiter JJ. The use of a dynamic hinged external fixator for complex, acute elbow instability. Trans Orthop Trauma Assoc 1996;274-275.

17. Milne AD, Chess DG, Johnson JA, King GJW. Accuracy of an electromagnetic tracking device: a study of the optimal operating range and metal interference. J Biomech 1996;29:791-793.

18. Morrey BF, Chao EYS, Hui FC. Biomechanical study of the elbow following excision of the radial head. J Bone Joint Surg [Am] 1979; 61A:63-68

19. Morrey BF, Askew LJ, An KN, Chao EYS. A biomechanical study of normal functional elbow motion. J Bone Joint Surg 1981;63A: 872-877.

20. Morrey BF, An KN. Articular and ligamentous contributions to the stability of the elbow joint. Am J Sports Med 1983;11:315-319.
21. Morrey BF, An KN. Functional anatomy of the ligaments of the elbow. Clin Orthop Rel Res 1985;201:84-90.

22. Morrey BF. Post-traumatic contracture of the elbow. J Bone Joint Surg [Am] 1990;72A:601-618.

23. Morrey BF, Tanaka S, An KN. Valgus stability of the elbow. A definition of primary and secondary constraints. Clin Orthop Rel Res 1991;265:187-195.

24. Morrey BF. Distraction arthroplasty. Clin Orthop Rel Res 1993; 293:46-54.

25. Morrey BF. Current concepts in the treatment of fractures of the radial head, the olecranon, and the coronoid. J Bone Joint Surg [Am] 1995;77A:316-327.

26. O'Driscoll SW, Horii E, Morrey BF, Carmichael SW. Anatomy of the ulnar part of the lateral collateral ligament of the elbow. Clin Anat 1992;5:293-303.

27. O'Driscoll SW, Morrey BF, Korinek BS, An KN. Elbow subluxation and dislocation. Clin Orthop Rel Res 1992;280:186-197.

28. Protzman RR. Dislocation of the elbow joint. J Bone Joint Surg [Am] 1978;60A:539-541

29. Regan WD, Korinek BS, Morrey BF, An KN. Biomechanical study of ligaments around the elbow joint. Clin Orthop Rel Res 1991; 271:170-179.

30. Schwab GH, Bennet JB, Woods GW, Tullos HS. Biomechanics of elbow instability: the role of the medial collateral ligament. Clin Orthop Rel Res 1980;146:42-51.

31. Shiba R, Sorbie C, Siu DW, Byrant JT, Cooke TDV, Wevers HW. Geometry of the humeroulnar joint. J Orthop Res 1988;6:897906.

32. Sojbjerg JO, Ovesen MDJ, Gundorf CE. The stability of the elbow following excision of the radial head and transsection of the annular ligament. Arch Orthop Trauma Surg 1987;106:248-250.

33. Sojbjerg JO, Ovesen MDJ, Nielson S. Experimental elbow instability after transsection of the medial collateral ligament. Clin Orthop Rel Res 1987;218:186-190.

34. Volkov MV, Oganesian OV. Restoration of function in the knee and elbow with a hinge-distractor apparatus. J Bone Joint Surg [Am] 1975;57A:591-600.

35. Werner FW, An KN. Biomechanics of the elbow and forearm. Hand Clin 1994;10:357-373.

36. Wyrsch RB, Seiler JG, Weikert DR, Murray D, Wyrick J, Stern PJ Early experience with the compass elbow hinge: a retrospective review. Orthop Trans 1997;21:442. 